\title{
REVIEW
}

\section{Post-Marketing Assessment of Neuropsychiatric Adverse Events in Influenza Patients Treated with Oseltamivir: An Updated Review}

Stephen Toovey - Eric P. Prinssen - Craig R. Rayner - Bharat T. Thakrar · Regina Dutkowski - Annette Koerner • Tom Chu $\cdot$ Alexandra Sirzen-Zelenskaya $\cdot$ Markus Britschgi $\cdot$ Sudhir Bansod $\cdot$ Barbara Donner

To view enhanced content go to www.advancesintherapy.com Received: June 8, 2012 / Published online: October 2, 2012

(C) The Author(s) 2012. This article is published with open access at Springerlink.com

\section{ABSTRACT}

A 2008 review by our group concluded that the risk of neuropsychiatric adverse events (NPAEs) in influenza patients was not increased by oseltamivir exposure, and did not identify any mechanism by which oseltamivir or its metabolites could cause or worsen such events. The current article reviews new information on this topic. Between September 16, 2007 and May 15, 2010, 1,805 spontaneously-reported NPAEs were identified in 1,330 patients receiving oseltamivir: 767 (42.5\%)

S. Toovey $(\varangle) \cdot$ E. P. Prinssen · B. T. Thakrar · A. Koerner · A. Sirzen-Zelenskaya · M. Britschgi · S. Bansod · B. Donner F. Hoffmann-La Roche Ltd., PBMT Bldg. 74/3O Z01.05, CH-4070, Basel, Switzerland

e-mail: malaria@sunrise.ch

C. R. Rayner

Roche Products Pty. Ltd., Melbourne, Australia

R. Dutkowski · T. Chu

Hoffmann-La Roche, Inc., Nutley, NJ, USA

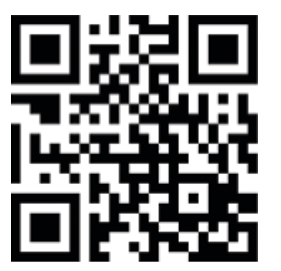

Enhanced content for Advances in Therapy articles is available on the journal web site: www.advancesintherapy.com from Japan, 296 (16.4\%) from the USA, and 742 (41.1\%) from other countries. NPAEs were more common in children: 1,072 (59.4\%) events were in those aged $\leq 16$ years. NPAEs often occurred within $48 \mathrm{~h}$ of treatment initiation (953 events; $52.8 \%$ ). Nearly half of the events were serious in nature $(838 ; 46.4 \%)$. The three largest categories of events were abnormal behavior (457 events, 25.3\%), miscellaneous psychiatric events $(370 ; 20.5 \%)$, and delusions/perceptual disturbances (316 events, 17.5\%). A total of 1,545 events $(85.6 \%)$ in eight different categories were considered to be delirium or delirium-like. Twenty-eight suicide-related events were reported. A US healthcare claims database analysis showed that the risk of NPAEs in 7,798 oseltamivir-treated patients was no higher than that in 10,411 patients not on antivirals, but a study on oseltamivir and abnormal behavior in Japan was less conclusive. NPAE frequency in oseltamivir-exposed Japanese and Taiwanese children with influenza was the same as in unexposed children. New analysis of the UK General Practice Research Database showed that the relative adjusted risk of NPAEs in influenza patients was 2.18-times higher than in the general population. Other epidemiology studies report frequent occurrence of encephalitis and similar disorders in influenza patients independently of 
oseltamivir exposure. The new data support the findings of the original assessment. Evidence suggests that influenza-related encephalopathies are caused by influenza-induced inflammatory responses, but more work is needed to confirm the underlying mechanisms.

Supplementary material is available on SpringerLink.com.

Keywords: Adverse events; Central nervous system; Influenza; Neuropsychiatric; Oseltamivir; Safety; Tolerability

\section{INTRODUCTION}

In December 2008, our group reported an assessment of influenza-associated neuropsychiatric adverse events (NPAEs) in patients who had received oseltamivir [1]. The article described the steps taken by Roche to assess the prevalence and genesis of such events by reviewing a wide variety of data, including surveillance of post-marketing spontaneous adverse event reports and results of preclinical and clinical studies. NPAEs had been known to be associated with influenza infection in Japanese patients before the introduction of oseltamivir [2-4], but reports, principally on Japanese patients during 2005/2006, had suggested that NPAEs might be associated with oseltamivir treatment [5]. The conclusion of the 2008 review was that the available data did not indicate that the risk of NPAEs in influenza patients was increased by exposure to oseltamivir, and that there was no obvious mechanism by which oseltamivir or its active metabolite could cause or worsen such events.

The purpose of the current article is to update the 2008 review, by reporting additional information generated since the first article was written. For ease of comparison, this update uses the same structure as the original article, but only reports new data. Additional published information was identified by a literature search, the scope of which was wider than that of the 2008 review - its aim was to identify reports of NPAEs in patients with influenza irrespective of treatment with oseltamivir or other antivirals. Medline and EMBASE databases were searched on January 11, 2011 for publications in English that contained the term influenza (and similar constructions such as "flu") and at least one of 34 terms relating to central nervous system (CNS) disorders and symptoms, such as encephalitis, loss or depressed level of consciousness, delirium, convulsion, hallucination, and abnormal behavior. This identified 493 publications, abstracts of which were independently screened by two experts to exclude those not relevant to influenza-associated CNS disorders. The 94 publications remaining after screening are summarized in the appropriate sections below, together with other articles that were found during the screening and reviewing process and deemed to be of relevance.

\section{CLINICAL SAFETY ASSESSMENT}

\section{Post-Marketing Reports of NPAEs in the Roche Global Safety Database}

The 2008 review presented an analysis of spontaneously reported NPAEs in the Roche Global Safety Database from 1999 to September 15,2007 . A total of 3,051 events were reported for 2,466 patients. To briefly recapitulate, the Roche database includes all events reported by its affiliates worldwide and by Chugai Pharmaceuticals, Roche's Japanese marketing partner. The case definition of NPAEs comprised 51 MedDRA high-level terms (106 associated preferred terms) in three System Organ Classes (nervous system disorders, psychiatric disorders, 
and accidents/injuries). In the 2008 review, all NPAEs were grouped into 13 categories to align with descriptions in the 9th edition of International Classification of Diseases (Table 1), and this grouping was repeated for the current update. A detailed case review was undertaken for all serious NPAEs.

This supplementary analysis of NPAEs covers the 32-month period from September 16, 2007 to May 15, 2010, which included the pandemic influenza outbreaks in the southern and northern hemispheres in 2009/2010 and previous seasonal influenza outbreaks. During this period, 1,805 NPAEs were spontaneously reported in 1,330 patients, with 1,072 of these events (59.4\%) occurring in children $\leq 16$ years (Table 1 ). The proportion attributable to children was somewhat lower than that reported in the original review (72.7\%). However, the difference in distribution by country compared with the original review is substantial: Japanese patients accounted for $42.5 \%$ of all events in the current analysis, a much smaller proportion than previously (Japan accounted for $90.9 \%$ of NPAEs in the first review). In the current analysis, patients from the USA and from the rest of the world (ROW) accounted for $16.4 \%$ and $41.1 \%$ of events, respectively (up from $6.2 \%$ and $2.9 \%$ in the original review). Comparing this supplementary analysis with the original of 2008, crude reporting rates per million filled

Table 1 Summary of post-marketing neuropsychiatric events in children (aged $\leq 16$ years) and adults receiving oseltamivir for influenza treatment or prophylaxis from September 16, 2007 to May 15, 2010

\begin{tabular}{|c|c|c|c|c|c|c|}
\hline & \multicolumn{3}{|c|}{ All adverse events } & \multicolumn{3}{|c|}{ Serious adverse events } \\
\hline & $\begin{array}{l}\text { All } \\
n(\%)\end{array}$ & $\begin{array}{l}\text { Adults } \\
n(\%)\end{array}$ & $\begin{array}{l}\text { Children } \\
n(\%)\end{array}$ & $\begin{array}{l}\text { All } \\
n(\%)\end{array}$ & $\begin{array}{l}\text { Adults } \\
n(\%)\end{array}$ & $\begin{array}{l}\text { Children } \\
n(\%)\end{array}$ \\
\hline Abnormal behavior & $457(25)$ & $76(10)$ & $381(36)$ & $170(20)$ & $31(9)$ & $139(28)$ \\
\hline Accident/injury & $27(1)$ & $20(3)$ & $7(1)$ & $18(2)$ & $14(4)$ & $4(1)$ \\
\hline Cognition disturbance & $171(9)$ & $97(13)$ & $74(7)$ & $93(11)$ & $46(13)$ & $47(10)$ \\
\hline Convulsions & $104(6)$ & $48(7)$ & $56(5)$ & $88(11)$ & $39(11)$ & $49(10)$ \\
\hline Delirium & $79(4)$ & $27(4)$ & $52(5)$ & $65(8)$ & $23(7)$ & $42(9)$ \\
\hline Delusions/perceptual disturbance & $316(18)$ & $121(17)$ & $195(18)$ & $164(20)$ & $70(20)$ & $94(19)$ \\
\hline Depressed level of consciousness & $32(2)$ & $17(2)$ & $15(1)$ & $15(2)$ & $12(3)$ & $3(1)$ \\
\hline Encephalitis & $27(1)$ & $10(1)$ & $17(2)$ & $27(3)$ & $10(3)$ & $17(3)$ \\
\hline Loss of consciousness & $37(2)$ & $29(4)$ & $8(1)$ & $19(2)$ & $15(4)$ & $4(1)$ \\
\hline Miscellaneous psychiatric & $370(20)$ & $191(26)$ & $179(17)$ & $107(13)$ & $50(14)$ & $57(12)$ \\
\hline Panic attack & $19(1)$ & $11(2)$ & $8(1)$ & $9(1)$ & $6(2)$ & $3(1)$ \\
\hline Parasomnia & $138(8)$ & $63(9)$ & $75(7)$ & $35(4)$ & $10(3)$ & $25(5)$ \\
\hline Suicidal events & $28(2)$ & $23(3)$ & $5(0)$ & $28(3)$ & $23(7)$ & $5(1)$ \\
\hline Total number of events & 1,805 & 733 & 1,072 & 838 & 349 & 489 \\
\hline Total number of patients & 1,330 & 523 & 807 & 603 & 249 & 354 \\
\hline
\end{tabular}

MedDRA terms and associated preferred terms in three classes (nervous system disorders, psychiatric disorders, and accidents/injuries) are grouped into 13 categories 
Table 2 Crude reporting rate of post-marketing neuropsychiatric events (events per one million oseltamivir prescriptions), from September 16,2007 to May 15, 2010

\begin{tabular}{llll}
\hline Country & Number of NPAEs & $\begin{array}{l}\text { Prescriptions } \\
(\text { million })\end{array}$ & $\begin{array}{l}\text { Event rate per million } \\
\text { prescriptions }\end{array}$ \\
\hline Japan & 767 & 14.07 & 54.5 \\
USA & 296 & 12.03 & 24.6 \\
Other countries & 742 & 3.53 & 210.2 \\
\hline
\end{tabular}

NPAEs neuropsychiatric adverse events

prescriptions (Table 2) fell markedly in Japan, from 127 to 55 , whereas in the ROW, the rate rose more than twofold (210; original review, 92). In the USA, the rate fell slightly from 27 to 25 . The rate increase in the ROW in 2009/2010 was driven by public health responses to the pandemic, notably in the UK, which accounted for $36 \%$ of all adverse events reported to Roche from May 1 to December 31, 2009 [6]. A new portal for telephone and internet-based reporting of adverse events in the UK was introduced by the Medicines and Healthcare Products Regulatory Agency on July 23, 2009 and operated until February 11, 2010.

As in the original review, more events were reported by men than women (957 vs. 737); for the remaining 111 events, the patient's gender was unknown. For 663 events, latency was unknown, and in the remaining 1,142 events, the majority (953 [83.4\%]) occurred within 2 days of starting oseltamivir treatment. This proportion was a little higher than reported in the original review (i.e., $77.6 \%$ of events). Only 39 patients reported NPAEs (56 events) while using oseltamivir for influenza prophylaxis (see below) - the remaining patients used it for influenza treatment. In all, 838 events (in 603 patients) were classified as serious (SAEs) - as a proportion of the total (46.4\%), this was considerably higher than in the original review (24\%). For 601 events, the outcome was not reported; of the remaining 1,204 events,
995 (82.6\%) improved or resolved, a slightly higher proportion than in the original review (77.6\%). The reporting rate of deaths associated with NPAEs $(23 / 1,805$ [1.3\%]) was a little higher than in the previous analysis $(0.6 \%) ; 18$ of the deaths were related to encephalitis or encephalopathy.

The most common category of NPAEs was abnormal behavior, which accounted for 457 (25.3\%) events across all age groups, the next most common categories being miscellaneous psychiatric events (370 events; 20.5\%) and delusions and perceptual disturbances (316 events; $17.5 \%$ ). These three categories constituted $63 \%$ of all NPAEs, a slightly lower proportion than in the original review (72\%), in which abnormal behavior was also the most common category (38.0\% of all events).

All information in the Roche safety database about the NPAE was used to assess the relationship between the event in question and accompanying fever and diagnosis of influenza in those patients who had taken oseltamivir as influenza treatment (not as prophylaxis). Information on whether fever was present or not at the time of the NPAE was missing for 289/457 (63\%) younger patients (aged $\leq 16$ years) and for $244 / 289$ (84\%) adult patients. In patients for whom data were available, fever was present in 121 of $168(72 \%)$ younger patients and in 29 of 45 adults (64\%). Timing of NPAEs relative to the influenza diagnosis was recorded in 34\% of adult cases and $45 \%$ of children. The event 
occurred within 2 days of the diagnosis in 129 of 205 (63\%) children and 31 of 98 (32\%) adults, which is consistent with the information on latency of event relative to treatment initiation as described above.

\section{Delirium and Delirium-Like Symptoms}

For the majority of NPAEs in adults and children $(1,545 / 1,805 ; 85.6 \%$; Table 1$)$, delirium and delirium-like symptoms were reported. All events in the following seven categories were associated with such symptoms: delirium, perceptual disturbances, cognition disturbances, depressed level of consciousness, panic attacks, parasomnia, and abnormal behavior. In addition, most of the events in the miscellaneous psychiatric events category were associated with delirium and delirium-like symptoms. Of the 1,545 events of this type, 976 were reported in children (91\% of all events in children) and 569 in adults (78\%), and the proportions classified as SAEs were similar in children and adults: 410/976 (42\%) and 226/569 (40\%), respectively (Table 3). However, the association of delirium and delirium-like symptoms with NPAE categories in children was different to that in adults. In children, they were most commonly associated with abnormal behavior (39\%); delusions and perceptual disturbances accounted for $20 \%$ and miscellaneous psychiatric events for $18 \%$ of these symptoms. In adults, however, the strongest association was with miscellaneous psychiatric events (32\%; Table 3).

\section{Accidents and Injuries}

All reports of accidents and injuries in the safety database were reviewed, whether or not they were associated with NPAEs. Twentyseven events were reported in 23 individuals (seven events in six children); 18 events in 15 individuals were serious; one of these events

Table 3 Categorization of delirium and delirium-like events (including serious adverse events) in influenza patients who received oseltamivir, reported from September 16, 2007 to May 15, 2010

\begin{tabular}{lllll}
\hline & $\begin{array}{l}\text { Adverse events } \\
n(\%)\end{array}$ & & \multicolumn{2}{l}{$\begin{array}{l}\text { Number of SAEs/number of patients with } \\
\text { SAEs }\end{array}$} \\
Category & Adults & Children $^{\mathrm{b}}$ & Adults & Children $^{\mathrm{b}}$ \\
\hline Delirium & $23(4)$ & $52(5)$ & $19 / 19$ & $42 / 42$ \\
Delusions and perceptual & $115(20)$ & $194(20)$ & $68 / 63$ & $93 / 88$ \\
disturbances & & & \\
Cognition disturbances & $88(15)$ & $73(7)$ & $38 / 33$ & $47 / 37$ \\
Parasomnia & $61(11)$ & $75(8)$ & $10 / 9$ & $25 / 25$ \\
Abnormal behavior & $73(13)$ & $381(39)$ & $29 / 29$ & $139 / 138$ \\
Depressed level of consciousness & $17(3)$ & $15(2)$ & $12 / 12$ & $3 / 3$ \\
Panic attacks & $10(2)$ & $7(1)$ & $6 / 6$ & $3 / 3$ \\
Miscellaneous psychiatric & $182(32)$ & $179(18)$ & $44 / 35$ & $57 / 44$ \\
Total & $569(100)$ & $976(100)$ & $226 / 206$ & $410 / 380$ \\
\hline $\begin{array}{l}\text { SAEs serious adverse events } \\
\text { a Serious and nonserious }\end{array}$ & & & & \\
b Aged $\leq 16$ years & & & & \\
\hline
\end{tabular}


was in a patient receiving prophylaxis. The most common events were falls and contusions. In 10 of the 14 influenza patients treated with oseltamivir who had SAEs, NPAEs such as loss of consciousness, confusion, dizziness, and hallucinations were associated with the accident or injury. In nine of 12 SAEs for which information on timing of the event was available, the event occurred early in the disease course (i.e., within $48 \mathrm{~h}$ of taking oseltamivir). For 10 of the 14 serious cases, information was insufficient to assess any association between oseltamivir exposure and the accident or injury. In two cases, comedication and medical history were possible confounders, and in two others, influenza was the only possible confounder. Information on timing of the serious accident or injury relative to the development of fever and other symptoms was only available for two cases: the first of these (contusion) happened within 1 day of the start of fever and the other (road traffic accident) happened 6 days after the onset of pyrexia.

\section{Suicide-Related Events}

The original review reported incidence of suicide-related events in patients exposed to oseltamivir. An extended search was performed of the Roche Global Safety Database for spontaneous reports of this type of adverse event from the date of the first market authorization up to September 17, 2009. A total of 39 cases were found, the most common preferred terms for the events being suicidal ideation (18) and suicidal attempt (8), with five cases of completed suicide. The other events were self-injurious behavior (5), intentional self-injury (2), and self-injurious ideation (1). In eight of the 39 cases of suicide-related events, information on medical history was insufficient; in 12 of the remaining 31 cases, psychiatric disorders that may have precipitated the event were present. Other NPAEs were associated with $26(67 \%)$ cases - the symptoms reported included anxiety, depression, abnormal behavior, sleep disorder, hallucinations, phobia, paranoia, and delusion. In nine cases, no associated NPAEs were reported, and information was lacking in the remaining four cases.

The crude reporting rates for suicide-related events and completed suicides, respectively, in patients exposed to oseltamivir (patient exposure of approximately 64.7 million patients) were 0.060 and 0.008 events per 100,000 patients. The reporting rate of completed suicide is markedly lower than published rates for completed suicides in the general population, which range from 15 in the UK to 51 in Japan (per 100,000 persons). The available data are therefore not compatible with oseltamivir having a causal role in suicidal ideation and/or suicide in influenza patients.

\section{NPAEs in Patients on Oseltamivir Prophylaxis}

The analysis of the three seasons from 2007/2008 to $2009 / 2010$ found that of 1,330 patients who reported NPAEs on oseltamivir, 39 (2.9\%) were taking it for prophylaxis. This fraction is higher than in the original review $(19 / 2,466 ; 0.8 \%)$. A case review of the 39 prophylaxis patients, in whom a total of 56 NPAEs were reported, showed that in 22 (56\%) cases, there were alternative explanations for the event. In the 17 other cases (44\%), insufficient information was available for analysis of causality. A causal link between oseltamivir prophylaxis and these events could not be established.

\section{NPAES IN PROSPECTIVE CLINICAL TRIALS OF OSELTAMIVIR}

The original review included a summary of NPAEs reported during prospective clinical studies in 
the Roche database, comparing oseltamivir with placebo in both treatment and prophylaxis indications. In treatment studies, reporting rates for oseltamivir $(0.5 \%)$ and placebo recipients $(0.6 \%)$ were very similar [1], and no new treatment studies have been performed since then. However, additional prophylaxis studies have been completed, increasing the cumulative total of patients exposed to oseltamivir in this indication to 2,856, of whom 1,902 took part in randomized, blinded studies. The updated incidence rates are similar to those in the previous review: significantly fewer oseltamivir patients $(12 / 1,902 ; 0.6 \%)$ reported NPAEs than placebo recipients $(20 / 1,365 ; 1.5 \%$; $P<0.05$, two-sided Fisher's exact test).

\section{NPAES IN RETROSPECTIVE SURVEILLANCE STUDIES}

In the 2008 review, results of three retrospective observational studies were summarized. All three used health claims data from US databases to compare NPAE incidence in a cohort of patients receiving oseltamivir and a cohort receiving no antiviral treatment, and showed that the use of oseltamivir was not associated with a higher risk of NPAEs [1].

The third of these retrospective studies, an analysis of data from two databases (MedStat [MS] and United Healthcare [UH]), has been extended to include a 6th year of claims data, bringing the number of patients per cohort to 106,487 and 102,591 , respectively, in the two databases. Patients in the two cohorts (aged $>1$ year and diagnosed with influenza between October 1, 2001 and September 30, 2007) were matched using propensity scores, and incidence of NPAEs was compared across 12 categories (the same as those used for the analysis of spontaneously reported NPAEs, omitting accidents and injuries). The analysis of this extended dataset produced results very similar to those reported in the 2008 review [1]. For patients of all ages, incidence rates for most of the NPAE categories analyzed were lower in the oseltamivir cohort than the no-antiviral cohort, and for some categories, the difference was significant. Oseltamivir users did not have a significantly higher risk of an NPAE in any category. For all NPAE categories combined, the risk of an event was significantly lower with oseltamivir than with no antiviral: total incidence rates were $0.62 \%$ and $0.97 \%$, respectively, in $\mathrm{UH}$ (odds ratio $[\mathrm{OR}]=0.64 ; 95 \%$ confidence interval [CI]: 0.58-0.71) and 0.23\% and $0.33 \%$, respectively, in MS (OR $=0.72$; 95\% CI: $0.61-0.85)$. In patients $\leq 16$ years old (approximately 40,000 per cohort), there was no significant difference between cohorts in NPAE risk. The total incidence of NPAEs was lower in this age group than in the whole cohort (all ages): $0.37 \%$ (oseltamivir) and $0.45 \%$ (no antiviral) in UH and $0.20 \%$ and $0.19 \%$, respectively, in MS. The extended analysis does not support a higher rate of NPAEs among oseltamivir users than nonusers, and suggests that the drug might have a protective effect, particularly in adult patients.

Two observational studies published in 2009 also found no evidence that oseltamivir treatment increases the rate of influenza-associated NPAEs. The results of the first study, on patients $\geq 1$ year old in the UH database [7], were reported in the 2008 review [1], as they had been presented at a scientific meeting in 2007. In summary, that study found a significantly lower incidence of NPAEs in the oseltamivir cohort than the no-antiviral cohort, a difference that applied to analyses of all events, major events, and events specific to CNS disorders. In patients $\leq 17$ years old, incidence rates in the two cohorts ( $n=20,250$ and 80,900 respectively) were not significantly different [7].

A new study, not analyzed in the first review, was undertaken in children and adolescents 
(aged 1-21 years) with an influenza diagnosis, using data from the US Department of Defense's TRICARE database, which contains healthcare information on over 9 million beneficiaries [8]. The incidence of all NPAEs was significantly lower in the oseltamivir cohort $(n=7,798)$ than the no-antiviral cohort $(n=10,411)$, with rates of $3.0 \%$ and $3.8 \%$ respectively $(P<0.05)$. Unlike the two analyses described above, groups were not matched for propensity scores, but a logistic regression analysis that adjusted for this factor found a significantly lower risk of events in the oseltamivir cohort $(\mathrm{OR}=0.82 ; 95 \%$ CI: 0.69-0.96). The authors also found the risk of an NPAE rose consistently with increasing age, whether or not the patient was treated. The range of events described as NPAEs in this study was similar to that in the Roche safety database analysis and in the combined MS + UH study reported in the review of Toovey et al. [1], but did also include stroke.

The results of a surveillance study conducted for the Japanese Ministry of Health, Labor, and Welfare during the $2006 / 2007$ season in 10,017 children and adolescents with influenza aged $<18$ years, of whom 7,586 received oseltamivir [9], have prompted extensive discussion. The author concluded that there was no correlation between treatment and abnormal behavior, but also noted that interpretation was complicated by limitations in the study method, and called for a case-control study to confirm the findings. According to a conditional logistic model, abnormal behavior as a whole was significantly less likely during treatment with oseltamivir $(\mathrm{OR}=0.62 ; 95 \% \mathrm{CI}: 0.51-0.76)$, although for abnormal behavior that carried a significant risk of injury or death, no such treatment advantage was shown $(\mathrm{OR}=1.25$; 95\% CI: 0.37-4.23). Other researchers subsequently criticized the reallocation of patients from the treated to the untreated groups in this study: the re-calculation by Yorifuji et al. [10] gave rates of abnormal behavior of $13.0 \%$ and $8.8 \%$ in treated and untreated groups, respectively (risk ratio $=1.48$; 95\% CI: 1.28-1.72), suggesting that oseltamivir use was positively associated with such behavior. However, since this recalculation itself introduces potential biases, as pointed out by Peng and Robinson [11], the findings of the original surveillance study remain difficult to interpret.

In a retrospective chart review in US infants aged $<1$ year with suspected or confirmed influenza [12], abnormal neurological events occurred in 19 of 115 (16.5\%) infants who received oseltamivir (doses of up to $7.0 \mathrm{mg} / \mathrm{kg}$ ) and in a similar proportion of infants who received amantadine or rimantadine (17/65; $26.2 \% ; P=0.13)$. The most common event $(13 / 22$ and $13 / 18$, respectively) was recorded as "irritable", which suggests a mood disorder rather than a neurological one; two seizures occurred, one in each group [12].

\section{REPORTS OF NPAES IN INFLUENZA PATIENTS AND RELATIONSHIP TO OSELTAMIVIR TREATMENT}

In the 2008 review, five reports from Japan and Taiwan on influenza patients aged $\leq 18$ years indicated that NPAEs occurring in influenza patients were similar whether or not they are exposed to oseltamivir, and that many events occur before treatment initiation [1]. Since then, a paper by Hama [13] was published that presents details of adverse events in eight Japanese patients who were prescribed oseltamivir, the events typically being of sudden onset. The events resulted in five deaths, three during sleep, and two from accidents associated with abnormal behavior. All these cases are recorded in the Roche safety database, and review of the cases found no conclusive evidence that oseltamivir had caused the NPAEs or the deaths. 
The great majority of data in other recently published studies does not support the hypothesis that oseltamivir is the cause of influenza-related NPAEs. A survey of 2,651 young patients (aged $<18$ years) admitted to hospital in Taiwan with influenza found that 74 (2.8\%) had CNS complications such as encephalopathy or seizures at hospital presentation [14]. No patient received oseltamivir before symptom onset, but in the 17 who received oseltamivir after admission, outcomes were not significantly different from the 57 untreated patients. In a smaller Japanese study of 22 children and adolescents hospitalized with influenza and with abnormal behavior, such as hallucinations and meaningless speech, the clinical course and outcomes in children who had CNS symptoms after oseltamivir treatment were similar to those whose symptoms preceded treatment [15]. In another Japanese study of 11 children who had delirious behavior within 3 days of the onset of influenza, seven had received no antiviral therapy, two patients had received oseltamivir, and two had received zanamivir, which is poorly bioavailable systemically [16].

The literature search identified case reports or case series describing NPAEs in 62 influenza patients exposed to oseltamivir - the most common events were seizures, encephalitis, and altered consciousness, and were mainly reported in children and adolescents. For 10 of these patients, the timing of oseltamivir treatment relative to the development of the events was not clearly described; eight of 10 patients recovered without sequelae [17-20].

Of 52 patients in whom the timing of oseltamivir administration relative to the NPAE was documented, most (38) received oseltamivir after the event had occurred, thus excluding the possibility of a causal association between oseltamivir and the event. Thirteen of the patients were described in single case reports, and the other 25 were from 10 case series [17, 18, 21-41]. In the other 14 patients (eight from single case reports and six from five case series), the NPAE appeared or worsened after exposure to oseltamivir [22, 30, $34,39,41-49]$. One patient died, a 4-year-old girl with chronic renal failure: she started oseltamivir on admission to hospital, 3 days after onset of influenza symptoms, and developed seizures, respiratory arrest, and coma the next day. Her death 1 day later was ascribed to influenza-associated acute necrotizing encephalopathy (ANE) [41]. Eleven other patients recovered after the NPAE (eight fully, three partially): eight completed their oseltamivir treatment, and three did not. In the two other patients, CNS complications were unlikely to be related to oseltamivir exposure as they developed 2-3 weeks after completion of oseltamivir treatment. The first of these patients had hypoxic brain injury after a prolonged period of intubation for hypoxemic respiratory failure and the second, who had been intubated for 20 days for bilateral pneumonia, developed agitation and Parkinsonian symptoms on being extubated [48, 49]. For three of the 14 patients with an NPAE after oseltamivir exposure, the authors considered that the NPAE was possibly related to oseltamivir. The first of these cases was a 35-year-old man who after taking three doses of oseltamivir (and within 2 days of the onset of influenza) developed a mild encephalopathy with a splenial lesion and raised cytokines; oseltamivir was discontinued [46]. The second case was a 15-year-old boy who developed confusion and agitation with electroencephalograms (EEG) slowing on day 5 of oseltamivir treatment and who improved rapidly after treatment had stopped [34]. The third case was a 17-year-old, who had a generalized seizure after two doses of oseltamivir and who recovered with no further seizures; he completed a 5-day course of oseltamivir [43]. As described in the following sections, raised cytokines, splenial lesions, EEG slowing, and 
seizures have all been reported in influenza patients who have not been treated with oseltamivir.

\section{EPIDEMIOLOGY OF ADVERSE CNS EFFECTS IN INFLUENZA PATIENTS}

In the original review, an analysis of patients in the UK General Practice Research Database (GPRD) over five influenza seasons (2001/2002 to $2005 / 2006$ ) provided evidence of an association between influenza itself and adverse CNS effects. This analysis has now been expanded to include data from patients diagnosed with influenza or influenza-like illness in two additional seasons (October 1, 2006 to March 31, 2007 and October 1, 2007 to March 31, 2008). The analysis grouped NPAEs into most of the same categories as the analysis of spontaneously reported NPAEs (omitting abnormal behavior, accidents and injuries and miscellaneous psychiatric events), and calculated incidence rates per 100 person months. The relative risk of an NPAE in the 30 days after diagnosis (adjusted for age, sex, and history of NPAEs) was found to be 2.18-times higher in 85,300 influenza patients $(11,297$ of whom were aged $\leq 16$ years) than in the total database population of 2,940,300 patients (95\% CI: 1.98-2.40). The relative risk of cognition disturbance, delusions and perceptual disturbances, convulsions, panic attack, and loss of, or depressed level of, consciousness was significantly higher in influenza patients than controls (by a factor of $1.38-4.20$ ). Only 82 of all the influenza patients analyzed received oseltamivir (nine of those aged $\leq 16$ years), none of whom had an NPAE [50].

More evidence illustrating the incidence of NPAEs in influenza patients comes from a prospective study by the National Institute of Infectious Diseases in Japan [51]. The study followed all patients at any medical institution during the 2007/2008 influenza season who had both influenza-like illness and serious abnormal behavior that could cause injury or death. Seventy-seven patients were affected by serious abnormal behavior in 2007/2008; the median age of patients exhibiting such behavior was 8 years (range 2-29 years), with 55 (71\%) being male. The behavior happened within 2 days of onset of fever in most patients (80\%), and mostly occurred immediately after waking (48/77; 62\%). Information on drugs taken (alone or in combination) was available for $59(77 \%)$ of the patients: $11(18 \%)$ were taking oseltamivir, nine (15\%) zanamivir, 27 (46\%) paracetamol, and 19 (32\%) took no drugs. The findings are further evidence that influenza associated NPAEs occur in the absence of oseltamivir, and that the general clinical picture is for emergence early in the course of the illness.

\section{CHILDREN AND ADOLESCENTS}

The literature search identified many reports of adverse CNS effects associated with influenza infection (both pandemic H1N1 2009 and seasonal strains), mainly in children. Some of these publications recorded abnormal behavior, but most of the events were acute encephalitis or encephalopathy, which are recognized neurological complications of influenza [1]. Some studies in series of young patients reveal more about the strength of association between influenza and NPAEs, how frequent such events are and their typical features, and what factors influence susceptibility and mortality.

In a 5-year survey of children admitted to Swedish hospitals with acute encephalitis of any origin, influenza was identified as the etiology for five of the 93 cases [52], although results of another Swedish hospital survey suggest that influenzaassociated encephalitis may not be readily or easily recognized [53]. A prospective registry study of 311 children admitted to Toronto hospitals 
with acute encephalitis or encephalopathy found that 22 (7\%) had influenza, 14 of whom had no other pathogens [54]. Abnormalities in EEG are a sensitive tool for measuring encephalitis, and abnormal slowing of EEG was recorded in many children with influenza-associated encephalopathy (IAE) or altered mental status [17, 22, 28, 54-57]. Seizures are also a common presenting symptom in children with IAE or altered mental status $[17,24,27,43,54,56]$. Febrile seizures were recorded in $20.6 \%$ of children hospitalized with influenza in a Chinese study [58]. Two other Chinese studies in hospitalized children with influenza reported an incidence of febrile seizures of $27.6 \%$ and approximately $18.0 \%$, and incidences of encephalopathy of 1.6 and $0.6 \%$, respectively $[59,60]$. In 13 children admitted to an intensive care unit in China with influenza over a 4 -year period, one had encephalitis [61]. Several recent reports on series of children admitted to hospitals with pandemic 2009 (H1N1) influenza record CNS manifestations (most commonly seizures and encephalopathy) as presenting symptoms. These typically affected $4-8 \%$ of cases [21, 28, 55, 62-65], but were more prevalent in children admitted to an intensive care unit or needing mechanical ventilation $[66,67]$.

The recent evidence also indicates differences in disease features between younger and older children who have influenza-related CNS complications. The Toronto study found neuroimaging abnormalities in five of seven children $<2$ years of age and in three of seven children aged $\geq 2$ years [54]. Also, in a survey of 472 Japanese children with IAE, significantly more of those aged $\leq 5$ years $(270 / 343 ; 79 \%)$ had convulsions than those aged 6-15 years (42/76; 55\%) [68]. The younger group in the latter study also had a higher incidence of hepatic dysfunction (notably mild elevation of aspartate aminotransferase levels), more lowdensity areas on brain computed tomography scans and significantly more sequelae [68]. Marked elevations of aspartate aminotransferase ( $\geq 500 \mathrm{IU} / \mathrm{mL}$ ) were one of four variables that significantly increased mortality risk in children aged $<15$ years with IAE, others being hyperglycemia, blood or protein in the urine, and diclofenac use [69].

In the studies mentioned above, most or all of the patients were infected with influenza A virus infections, which reflects the relative prevalence of type A and type B viruses. Two reports from Taiwan focused solely on CNS events in young patients with influenza B infections. In the larger study, a survey of 314 patients, 13 (4.1\%) patients (mean age 5.3 years) had encephalitis or encephalopathy, one of whom died [57], and in the other study, one of 87 patients aged $<18$ years (1.1\%) had encephalitis [70]. Influenza B infection was also diagnosed in a 14-year-old Japanese boy who injured himself falling from a window after symptoms of parasomnia, fever, and fatigue [71]. A single case of acute encephalopathy associated with influenza $\mathrm{C}$ infection was also reported, in a 2-year-old Japanese boy, although the outcome was not described [72].

Children with more severe influenza complications, in particular ANE, continue to be the subject of case reports and series. ANE can have other etiologies, and a retrospective report of 22 Japanese children with ANE found that 11 (50\%) influenza-associated cases had similar pathology to 11 (50\%) noninfluenza-associated cases, although brain stem lesions were more common in the former group [73]. Outcomes in this study were also similar, with four and three deaths (36\% and 27\%), respectively. The high morbidity and mortality rate in ANE is reflected in nine single case reports in children and adolescents aged $2-12$ years old: the condition was fatal in four children, and caused severe disablement in two of five who survived [29, 32, 33, 36, 38, 74-77]. An inherited susceptibility to ANE has been discovered, characterized by a 
mutation in the RAN binding protein 2 gene [78], and the clinical presentation of two patients thus affected has recently been reported [74]. Another case report documented a 3-year-old girl with marked neurological impairment resulting from IAE combined with cerebral vasculopathy and edema characteristic of posterior reversible encephalopathy syndrome [79].

\section{Adults}

In contrast to the experience in Asian countries, a survey of hospitalized influenza patients in Sweden found that those affected by encephalitis were mainly middle-aged men, although cases were rare (21 in 11.5 years) [53]. Adults also featured in other recent reports of influenzarelated CNS complications. A 2009 survey of hospitalized influenza patients in Singapore found mild NPAEs, such as seizures, in nine patients aged 15-57 years, six of whom were $\geq 18$ years old [19]. A Chinese case series described three adults aged $72-86$ years with acute encephalopathy [30], and in a Chilean case series of six influenza patients with CNS dysfunction, three were adults aged 38-65 years [34]. Four other recent case reports on adults describe severe acute hemorrhagic leukoencephalitis in a 40-year-old man [48], a 46-year-old woman with confusion and delirium [26], a 39-year-old woman with influenzarelated acute oculomotor nerve palsy [40], and a 65-year-old woman with altered mental status secondary to influenza-associated posterior reversible encephalopathy syndrome with cerebral vasculopathy [80]. In a fifth report case, a 22-year-old woman with severe pandemic H1N1 2009 influenza complicated by pneumonia was intubated and mechanically ventilated for 20 days. On extubation, she developed severe agitation, Parkinsonian symptoms such as tremor and mask-like face, and hypothalamic abnormality [49]. Whether influenza can trigger chronic neurological disorders such as Parkinson's disease is uncertain; a recent review that focused mainly on influenza pandemics found little or no supporting evidence for a link [81], and an analysis of the UK GPRD also found no association between influenza infections and subsequent development of Parkinson's disease, although the risk of Parkinsonian symptoms was significantly associated with influenza infection that occurred in the previous month and with the number of previous influenza episodes [82].

\section{PHARMACOKINETIC PROFILE OF OSELTAMIVIR IN THE CNS}

\section{CNS Exposure to Oseltamivir and its Metabolite in Animals}

The original review summarized data, mostly from animal studies, that show penetration of both oseltamivir and its carboxylate metabolite into the CNS to be very low [1], and the results of our nonclinical studies on CNS pharmacokinetics have since been published separately [83]. Additional data have since been reported from a pharmacokinetic study in adult mice, which mapped the distribution of radiolabelled oseltamivir and oseltamivir carboxylate after intravenous injection. This study found that the ratio of concentrations for brain-to-plasma were $0.07-0.14$ and $0.06-0.13$, respectively [84]. These results are in general agreement with those of preclinical studies that employed a variety of methods [83], and somewhat higher than the cerebrospinal fluid (CSF)-to-plasma ratios of 0.020.03 found in healthy volunteers [85].

\section{Regulation of Oseltamivir and Oseltamivir Carboxylate Levels in the CNS}

The influence of a range of transport systems on the disposition of oseltamivir and 
oseltamivir carboxylate in the CNS and their pharmacodynamic effect was described in the original review. It was noted that P-glycoprotein actively transports oseltamivir out of the CNS, but active transport of oseltamivir carboxylate through the blood-brain barrier (BBB) had not yet been reported [1]. Recently, experiments using knockout mice and cell culture models reported by Ose et al. [86] have demonstrated that organic anion transporter 3 (OAT3) and human multidrug resistance protein 4 (MRP4) play a role in the active transport of oseltamivir carboxylate out of the CNS, although the relevance to humans is unclear (see below). This group also showed that brain distribution of oseltamivir carboxylate in MRP4-/- mice was higher than in wild-type mice, but the distribution in OAT3-/- mice was very similar to that in wild-type mice. The authors speculated that OAT3 might also mediate transport in the opposite direction (into the CNS). The authors of this study concluded that oseltamivir carboxylate can cross the BBB, but its disposition in the brain is limited by active transport systems such as OAT3 and MRP4. The nature of the interaction between these transport systems is still unclear, including how impairment of one system, for example as a result of genetic variations, might be compensated by another system. Additionally, it was reported by Uchida et al. [87] that protein expression of MRP4 and OAT3 in the BBB is different in mice and humans, with human values being 8.1 and $>5.7$-times lower, respectively, than in mice. These data suggest that, in this case, animal data may have limited relevance to humans.

In another recently reported pharmacokinetic study, mice with lipopolysaccharide (LPS)induced inflammation, which increases the permeability of the BBB, had brain concentrations of oseltamivir and oseltamivir carboxylate that were 2 and 2.7-times those in control mice [88].
These limited increases in brain concentrations in the presence of LPS-induced inflammation are regarded as clinically irrelevant, as there are very large safety margins $[83,89]$.

\section{PHARMACODYNAMIC PROFILE IN THE CNS}

\section{Animal Studies}

Some recent publications suggest that oseltamivir may have a stimulant effect in the CNS. One study by a US group reported that oseltamivir carboxylate facilitated neuronal firing in ex vivo rat hippocampal slices [90]; however, in a well-controlled study that used a similar protocol, the authors were not able to replicate this finding [91]. A second study, by a Japanese group, reported that oseltamivir carboxylate enhanced neuronal synchronization in pyramidal cells from the rat hippocampus ex vivo [92]. Interpretation of observed abnormal behavior in rodent hippocampal models is not straightforward, as they were initially developed to study learning and epilepsy. The reported excitatory properties of oseltamivir in these models contrast with the findings of pharmacology studies that showed no binding of oseltamivir carboxylate to a large variety of host targets including dopaminergic and glutamatergic targets $[89,93]$. In addition, in vivo animal studies have failed to show excitatory effects of oseltamivir and its metabolite either in a proconvulsive rodent model, on modulation of hexobarbitalinduced sleeping in rodents, or on hyperactivity in rodents, even at very high doses [94]. The study described above, which showed neuro-excitatory properties of oseltamivir carboxylate in rat models, also reported enhancement of this effect by ethanol [90]. Oseltamivir carboxylate and ethanol also 
produced hypothermia and altered behavior (e.g., changes in righting reflex, although the authors acknowledged that the latter were difficult to interpret). In addition, synaptic transmission in hippocampal slices was shown to be facilitated by a combination of oseltamivir carboxylate, ephedrine, and caffeine [95]. The use of ethanol and stimulants in these studies was prompted by the observation that Japanese influenza patients sometimes take these agents in combination with oseltamivir. However, the doses of ethanol used in the first rodent study (3.3 $\mathrm{g} / \mathrm{kg}$ in vivo; $60 \mathrm{mmol} / \mathrm{L} \mathrm{ex}$ vivo) were excessively high and would be highly toxic or even fatal in humans. Similarly, the concentrations of oseltamivir and its metabolite used were much higher than extrapolated therapeutic concentrations [89]. The reports in the Roche safety database did not indicate any concomitant intake of ethanol or ethanolcontaining products (e.g., cough syrups) in children experiencing NPAEs.

\section{Effect on Sleep Parameters}

To investigate the effect of oseltamivir on sleep, a placebo-controlled polysomnography study was conducted in 31 healthy Japanese adults using a crossover design. Sleep parameters and EEGs were assessed for four consecutive nights, with oseltamivir being given on the 3rd and 4 th days (75 mg single evening dose on day 3; two $75 \mathrm{mg}$ doses [morning and evening] on day 4). Compared with placebo, the study showed no clinically relevant effect on EEGs, on sleep parameters, or on normal sleep behavior [96].

\section{GENETIC INFLUENCES}

As mentioned above, oseltamivir is hydrolyzed in the body by human carboxylesterases (HCEs) to the active metabolite oseltamivir carboxylate $[97,98]$.
The specific enzyme responsible for the hydrolysis is HCE1, found principally in the liver [99]. As reported in the original review, this enzyme has several naturally occurring polymorphic variants that hydrolyze substrate in vitro faster or slower than the wild-type enzyme [99], but the frequencies of these polymorphisms and how they affect oseltamivir hydrolysis in humans are not yet known. Since the original review, a new publication has reported two more variants of HCE1 with greatly reduced hydrolysis capacity in vitro [100], but the authors acknowledge that these mutations have limited clinical relevance, given their rarity. In their recent study on gene expression and in vitro hydrolytic capacity of HCE1 in liver microsome samples, Yang et al. [101] reported that the ratio of exposure to oseltamivir carboxylate in children to that in adults in vitro was approximately 0.15 . This contrasts with the findings of an in vitro study, which showed levels of HCE1 expression in liver fractions to be low only in neonates in their first month of life [102]; in children aged 1-9 years old, expression was approximately $80 \%$ of that in adults. That result is consistent with the value of approximately 50\% from an in vivo pharmacokinetic comparison [103]. Yang et al. also reported that the hydrolytic capacity of fetal microsomes in vitro, although very variable, was about $10 \%$ of the adult value [101]. A subsequent study reported on oseltamivir pharmacokinetics in 20 premature neonates (median gestational age, 27.5 weeks; chronological age, 1.5-17.5 weeks) who received prophylaxis after exposure to pandemic H1N1 [104]. This sparse data set shows that premature babies, like older infants, probably have sufficient capacity to metabolize oseltamivir.

Although the results of these two new studies imply that partial or complete failure of the hydrolytic capacity of HCEs could happen in certain individuals because of genetic or agerelated variation, the oseltamivir concentrations 
that might be produced by complete absence of hydrolysis have not been shown to be associated with NPAEs. This was described both in the original review and in a more recent paper reporting nonclinical studies of CNS pharmacokinetics [1, 83].

\section{OTHER POSSIBLE ASSOCIATIONS BETWEEN INFLUENZA AND CNS DYSFUNCTION}

It has previously been suggested that elevated levels of cytokines in the plasma are correlated with and possibly cause CNS disorders associated with influenza $[105,106]$. Several papers found in the literature search provide additional evidence for this hypothesis. In a group of children with confirmed influenza, those with mild neurological complications (febrile seizures in 17 and delirium in 10) were shown to have significantly higher serum interleukin (IL)-6 levels (medians, 31.2 and $42.3 \mathrm{pg} / \mathrm{mL}$ for delirium and febrile seizure groups, respectively) than those without complications $(15.4 \mathrm{pg} / \mathrm{mL})$; however, levels of serum tumor necrosis factor alpha (TNF-alpha) and soluble TNF receptor1 (sTNF-R1) were similar in the three groups [56]. Cytokine elevation was also noted in case reports of acute encephalopathy and ANE [30, 72, 76], and elevation of IL-6 was shown to mirror abnormal EEG changes in influenzainfected rats with induced hyperthermia [107]. Other workers have shown that LPSinduced brain lesions in mice are enhanced by pulmonary infection with influenza A virus [108], producing hypercytokinemia, microhemorrhage, and an increase in BBB permeability. Rats with acute peripheral inflammation exhibited a marked but reversible inflammatory response in the hippocampus, characterized by reactive microglia, increased TNF-alpha levels, and increased susceptibility to pentylenetetrazol-induced seizures [109]. It is not yet clear how hypercytokinemia triggers CNS changes, although one group recently showed that a rise in plasma TNF-alpha in mice triggered rises in brain levels of matrix metalloproteinase-9 and increases in BBB permeability [110]. Another mechanism through which elevation of cytokines in the plasma could trigger acute phase responses in the CNS has been suggested by Serrats et al. [111]. They showed that intravenous challenge with IL-1 activated perivascular cells in the cerebral vasculature, resulting in activation of the hypothalamic-pituitary axis and the febrile response system, despite a functional BBB; LPS challenge also activated this system, producing even stronger hypothalamic-pituitary axis activation and febrile response [111]. The upregulation of systemic immune pathways, whether by viral infection or other stimulus, is known to significantly affect CNS function in Alzheimer's disease and multiple sclerosis $[112,113]$, and cytokines may play an important role in the pathogenesis of depression [114] and sleep disorders [115].

Several groups report reversible splenial lesions of the corpus callosum in patients with IAE, possibly caused by intramyelinic edema or inflammatory cell influx, but the exact role played by such lesions in the pathogenesis of NPAEs is unclear. The splenial lesions may be accompanied by EEG abnormalities $[16,116]$ or raised cytokine levels [46], and may result in mild CNS disorders in some younger patients [117-119]. A 14-year-old boy treated with the inhaled topical agent zanamivir had neurological symptoms, such as dysarthria and dysphagia, associated with a reversible splenial lesion 5 days after influenza onset [119]. A speech disorder in a boy with influenza and a splenial lesion was attributed by the authors to dysarthria of nonneurological origin [120]. An isolated report of 
postinfluenza pneumocephalus in a 9-year-old boy causing delirium, convulsions, and coma suggested that this could be an under-recognized mechanism for CNS symptoms in patients with recurrent coughing or sneezing [121].

Two reports found in the literature search show that invasion of cells by influenza virus results in apoptosis [122, 123], but only one of these studies was undertaken in nerve cells. Apoptosis would be expected to result from inflammatory changes such as hypercytokinemia, but as the influenza virus and viral RNA are rarely found in the brain or CSF of patients with IAE, direct viral invasion of CNS cells would be unlikely to trigger apoptosis [3, 30, 124, 125]. In IAE, however, the peripheral blood and CSF concentrations of a number of cytokines and their receptors, including IL-6, IL-1beta and sTNF-R1, are not only raised but also related to severity [126]. CNS hypercytokinemia in the absence of direct CNS invasion is a possible mechanism to explain the pathogenesis of IAE.

Other recent literature reports have identified possible genetic risk factors for CNS dysfunction. A recent study by Neilson et al. [78] showed that missense mutations in RAN binding protein 2 increased susceptibility to ANE in both individuals and families; individuals with this gene mutation are predisposed to develop this condition after an environmental trigger such as influenza and other infections. Two Japanese groups have reported polymorphisms in the enzyme carnitine palmitoyltransferase II in patients with encephalopathy secondary to infectious disease $[127,128]$. In patients with IAE, who expressed variants of carnitine palmitoyltransferase II, energy metabolism in mitochondria was impaired, which could contribute to brain edema via increased BBB permeability [128]. These findings are in line with previous reports that carnitine metabolism was impaired in many patients with severe IAE [129], and that serum concentrations of the mitochondrial protein cytochrome $\mathrm{C}$ can predict the severity of encephalopathy [130]. Another metabolic mechanism that could be linked to CNS damage is the presence of organic acidemias, inherited metabolic disorders, which were found in a small proportion of children with IAE [131].

The neuro-inflammatory and systemic changes mentioned above such as cytokine induction and alterations in BBB integrity are compatible with the types of injury observed, as is the disruption of mitochondrial energy metabolism, which is accompanied by neuronal oxidative stress and dysfunction, or in some cases, apoptosis. It is possible that individuals prone to mitochondrial dysfunction might be more susceptible to neuronal dysfunction and apoptosis in the presence of influenza-induced fever or cytokines. This is an area where further research would be helpful.

\section{CONCLUSIONS}

The analysis of spontaneously reported NPAEs over the three consecutive influenza seasons to May 2010 confirms the results of the 2008 review. In $83 \%$ of NPAE cases, the onset of these events was within 2 days of both the influenza diagnosis and the start of oseltamivir treatment. These associations in timing are a logical consequence of the recommendation to start oseltamivir treatment within $48 \mathrm{~h}$ of symptom onset, but have also led to claims that the treatment could have caused NPAEs. However, NPAEs also coincided to a large degree with the occurrence of fever. A close temporal relationship between the diagnosis of influenza and the onset of NPAEs is also seen in cases without oseltamivir exposure, as well as in patients exposed to zanamivir, which is 
not systemically bioavailable, pointing to a disease-associated process as pathogenesis. This is supported by the findings of clinical reports from some Asian countries, by an expanded analysis of a large general practice database, and by the results of more large observational studies in the USA (in children, adolescents, and adults) that did not show NPAE incidence to be increased by oseltamivir administration.

Reports from recently published literature on influenza patients who had CNS complications and received oseltamivir, show that in the majority of cases, the adverse events happened or developed before drug administration, and were not exacerbated by subsequent administration. Mild encephalopathy was also reported in a patient treated with zanamivir, which is not absorbed systemically to significant levels [119]. In nine cases where events happened or worsened after oseltamivir treatment, six were not considered to be drug-related, and NPAEs in the other three included symptoms such as seizures and EEG slowing, which are known to be associated with influenza infection itself, as described earlier.

In the authors' analysis of accidents and injuries in the Roche safety database, most of the SAE cases had an association with an NPAE, but reports were not detailed enough for the relationship with oseltamivir exposure to be assessed. A survey of Japanese health insurance claims data in 140,800 influenza patients of all ages (of whom 51,505 received oseltamivir) found that a total of 97 injuries occurred in the 3 days after influenza diagnosis, and that the crude incidence of injuries was significantly lower in oseltamivir-treated patients $(4.7$ per 10,000$)$ than in untreated patients (8.2 per 10,000 ; $P=0.015)$ [132].

Our updated literature search found no evidence from animal pharmacology studies that oseltamivir can produce harmful CNS effects, even at doses markedly higher than those typically used in clinical practice. Studies completed since the initial review confirm that the penetration into the CNS of oseltamivir and its active metabolite is low in animals, and it is unlikely that changes in the disposition or metabolism of oseltamivir in the brain, whether caused by genetic polymorphisms or other means, can markedly increase exposure to the drug. Even if this were to occur, recent studies showing lack of interaction with relevant CNS targets $[89,93]$, lack of interference with sleep in humans [96], and lack of excitatory effects in animals [94] suggest there would be no functional implications of such changes.

As reviewed above, more evidence has been accumulated for influenza-induced inflammatory responses and metabolic derangement being the most likely causative mechanisms for influenzarelated neuropsychiatric dysfunction. Changes due to peripheral inflammation during influenza may induce neuro-inflammation and apoptosis in CNS tissue, and elevated levels of some cytokines continue to be observed in patients with neurological complications of influenza. Indeed, a recent article by two US neurologists proposes that "cytokine storms," resulting from dysregulation of the immune system, are the most likely mechanism to explain these conditions [133]. The additional surveillance data and published evidence on influenza patients treated with oseltamivir have still not furnished any evidence for a causative link between exposure to drug and generation or worsening of NPAEs.

\section{ACKNOWLEDGMENTS}

Dr. Toovey is the guarantor for this article, and takes responsibility for the integrity of the work as a whole. 
Conflict of Interest. All authors contributed to the design, conduct, analysis, and/or interpretation of at least one of the investigations reported herein, some of which were supported by Roche. All authors participated in the preparation, review, and approval of this article. All authors are employees (Drs. Prinssen, Rayner, Thakrar, Dutkowski, Koerner, Chu, Sirzen-Zelenskaya, Britschgi, and Donner) of or consultants (Drs. Toovey and Bansod) to Roche. Drs. Prinssen, Thakrar, Dutkowski, Sirzen-Zelenskaya, and Donner also have stock ownership options in Roche. Dr. Toovey has received honoraria from Roche. Support for third-party writing assistance for this manuscript was provided by Roche.

Open Access. This article is distributed under the terms of the Creative Commons Attribution Noncommercial License which permits any noncommercial use, distribution, and reproduction in any medium, provided the original author(s) and source are credited.

\section{REFERENCES}

1. Toovey S, Rayner C, Prinssen E, et al. Assessment of neuropsychiatric adverse events in influenza patients treated with oseltamivir: a comprehensive review. Drug Saf. 2008;31:1097-114.

2. Kasai T, Togashi T, Morishima T. Encephalopathy associated with influenza epidemics. Lancet. 2000;355:1558-9.

3. Morishima T, Togashi T, Yokota S, et al. Encephalitis and encephalopathy associated with an influenza epidemic in Japan. Clin Infect Dis. 2002;35:512-7.

4. Okabe N, Yamashita K, Taniguchi K, Inouye S. Influenza surveillance system of Japan and acute encephalitis and encephalopathy in the influenza season. Pediatr Int. 2000;42:187-91.

5. Food and Drug Administration (FDA). Meeting of the FDA Pediatric Advisory Committee. November 16, 2006. Available at: http://www.fda.gov/OHRMS/ DOCKETS/ac/oc06.htm. Accessed Sep 102012.
6. Donner B, Bader-Weder S, Schwarz R, Peng MM, Smith JR, Niranjan V. Safety profile of oseltamivir during the 2009 influenza pandemic. Pharmacoepidemiol Drug Saf. 2011;20:532-43.

7. Smith JR, Sacks S. Incidence of neuropsychiatric adverse events in influenza patients treated with oseltamivir or no antiviral treatment. Int J Clin Pract. 2009;63:596-605.

8. Casscells SW, Granger E, Kress AM, Linton A. The association between oseltamivir use and adverse neuropsychiatric outcomes among TRICARE beneficiaries, ages 1 through 21 years diagnosed with influenza. Int $\mathrm{J}$ Adolesc Med Health. 2009;21:79-89.

9. Hirota Y. Survey research on the emergence of influenza-associated symptoms. 2007/2008 research subteam report. 2009. Tokyo: MHLW Scientific Research Fund.

10. Yorifuji T, Suzuki E, Tsuda T. Oseltamivir and abnormal behaviors: true or not? Epidemiology. 2009;20:619-21.

11. Peng MM, Robinson NJ. Oseltamivir and abnormal behavior. Epidemiology. 2010;21:915-6.

12. Kimberlin DW, Shalabi M, Abzug MJ, et al. Safety of oseltamivir compared with the adamantanes in children less than 12 months of age. Pediatr Infect Dis J. 2010;29:195-8.

13. Hama R. Fatal neuropsychiatric adverse reactions to oseltamivir: case series and overview of causal relationship. Int J Risk Saf Med. 2008;20:5-36.

14. Huang YC, Li WC, Tsao KC, et al. Influenzaassociated central nervous system dysfunction in Taiwanese children: clinical characteristics and outcomes with and without administration of oseltamivir. Pediatr Infect Dis J. 2009;28:647-8.

15. Tanabe T, Hara K, Nakajima M, Shimakawa S, Tamai H. Oseltamivir treatment for children showing abnormal behavior during influenza virus infection. Brain Dev. 2010;32:440-4.

16. Takanashi J, Tada H, Kuroki H, Barkovich AJ. Delirious behavior in influenza is associated with a reversible splenial lesion. Brain Dev. 2009;31:423-6.

17. Baltagi SA, Shoykhet M, Felmet K, Kochanek PM, Bell MJ. Neurological sequelae of 2009 influenza A (H1N1) in children: a case series observed during a pandemic. Pediatr Crit Care Med. 2010;11:179-84. 
18. Rellosa N, Bloch KC, Shane AL, Debiasi RL. Neurologic manifestations of pediatric novel H1N1 influenza infection. Pediatr Infect Dis J. 2011;30:165-7.

19. Tan K, Prerna A, Leo YS. Surveillance of H1N1related neurological complications. Lancet Neurol. 2010;9:142-3.

20. Wiwanitkit V. Swine flu encephalitis: a comment on the first case in Thailand. J Neurol Sci. 2009;26:518-9.

21. Calitri C, Gabiano C, Garazzino S, et al. Clinical features of hospitalised children with 2009 H1N1 influenza virus infection. Eur J Pediatr. 2010;169:1511-5.

22. Centers for Disease Control and Prevention (CDC). Neurologic complications associated with novel influenza A (H1N1) virus infection in children - Dallas, Texas, May 2009. MMWR Morb Mortal Wkly Rep. 2009;58:773-8.

23. Chen YC, Lo CP, Chang TP. Novel influenza A (H1N1)-associated encephalopathy/encephalitis with severe neurological sequelae and unique image features - A case report. J Neurol Sci. 2010;298:110-3.

24. Citerio G, Sala F, Patruno A, et al. Influenza A (H1N1) encephalitis with severe intracranial hypertension. Minerva Anestesiol. 2010;76:459-62.

25. German-Diaz M, Pavo-Garcia R, Diaz-Diaz J, Giangaspro-Corradi E, Negreira-Cepeda S. Adolescent with neuropsychiatric symptoms associated with novel influenza A (H1N1) virus infection. Pediatr Infect Dis J. 2010;29:570-1.

26. Gonzalez BE, Brust DG. Novel influenza A (H1N1) presenting as an acute febrile encephalopathy in a mother and daughter. Clin Infect Dis. 2009;49:1966-7.

27. Haktanir A. MR imaging in novel influenza A(H1N1)-associated meningoencephalitis. AJNR Am J Neuroradiol. 2010;31:394-5.

28. Kedia S, Stroud B, Parsons J, et al. Pediatric neurological complications of 2009 pandemic influenza A (H1N1). Arch Neurol. 2011;68:455-62.

29. Kwong KL, Hiu H, Hui CM, Que TL. A tragic case of flu. HK J Paediatr. 2007;12:199-201.

30. Lee N, Wong CK, Chan PK, et al. Acute encephalopathy associated with influenza A infection in adults. Emerg Infect Dis. 2010;16:139-42.
31. Li X, Du Y. Neurologic complications associated with novel influenza A (H1N1) virus infection in Nanchong, Chengdu, November 2009. J Exp Clin Med. 2010;2:254-6.

32. Mariotti P, Iorio R, Frisullo G, et al. Acute necrotizing encephalopathy during novel influenza A (H1N1) virus infection. Ann Neurol. 2010;68:111-4.

33. Martin A, Reade EP. Acute necrotizing encephalopathy progressing to brain death in a pediatric patient with novel influenza A (H1N1) infection. Clin Infect Dis. 2010;50:e50-e52.

34. Noriega LM, Verdugo RJ, Araos R, et al. Pandemic influenza A (H1N1) 2009 with neurological manifestations, a case series. Influenza Other Respi Viruses. 2010;4:117-20.

35. O'Leary MF, Chappell JD, Stratton CW, et al. Complex febrile seizures followed by complete recovery in an infant with high-titer 2009 pandemic influenza A (H1N1) virus infection. J Clin Microbiol. 2010;48:3803-5.

36. Ormitti F, Ventura E, Summa A, Picetti E, Crisi G. Acute necrotizing encephalopathy in a child during the 2009 influenza $\mathrm{A}(\mathrm{H} 1 \mathrm{~N} 1)$ pandemia: MR imaging in diagnosis and follow-up. AJNR Am J Neuroradiol. 2010;31:396-400.

37. Samuel N, Attias O, Tatour S, Brik R. Novel influenza A (H1N1) and acute encephalitis in a child. Isr Med Assoc J. 2010;12:446-7.

38. Tada H, Takanashi JI, Terada H, Tajima K. Severe form of acute influenza encephalopathy with biphasic seizures and late reduced diffusion. Neuropediatrics. 2008;39:134-6.

39. Webster RI, Hazelton B, Suleiman J, et al. Severe encephalopathy with swine origin influenza A H1N1 infection in childhood: case reports. Neurology. 2010;74:1077-8.

40. Yamashita S, Kouzaki Y, Kawano R, Tokunaga M, Uchino M. Acute ophthalmoparesis accompanied with influenza A infection. Intern Med. 2008;47:1627-9.

41. Yildizdas D, Kendirli T, Arslankoylu AE, et al. Neurological complications of pandemic influenza (H1N1) in children. Eur J Pediatr. 2011;170:779-88.

42. Akins PT, Belko J, Uyeki TM, et al. H1N1 encephalitis with malignant edema and review of neurologic complications from influenza. Neurocrit Care. 2010;13:396-406. 
43. Choi SY, Jang SH, Kim JO, et al. Novel swine-origin influenza A (H1N1) viral encephalitis. Yonsei Med J. 2010;51:291-2.

44. Costiniuk CT, Le SN, Sell E, et al. Miller Fisher syndrome in a toddler with influenza A (pH1N1) infection. J Child Neurol. 2011;26:385-8.

45. Gadoth A, Aizenstein O, Mosek A. Influenza A/ H1N1 encephalitis. Neurology. 2010;75:666-7.

46. Kimura E, Okamoto S, Uchida Y, et al. A reversible lesion of the corpus callosum splenium with adult influenza-associated encephalitis/encephalopathy: a case report. J Med Case Reports. 2008;2:220.

47. Sanchez-Torrent L, Trivino-Rodriguez M, SueroToledano P, et al. Novel influenza A (H1N1) encephalitis in a 3-month-old infant. Infection. 2010;38:227-9.

48. Fugate JE, Lam EM, Rabinstein AA, Wijdicks EF. Acute hemorrhagic leukoencephalitis and hypoxic brain injury associated with H1N1 influenza. Arch Neurol. 2010;67:756-8.

49. Gonzalez-Duarte A, Magana ZL, Cantu BC, GarciaRamos G. Hypothalamic abnormalities and Parkinsonism associated with H1N1 influenza infection. J Neuroinflammation. 2010;7:47.

50. Data on file. Basel: Roche; 2010.

51. Okabe N. Research on Abnormal Behaviour Accompanying Influenza - Report of the 20082009 season. Available at: http://www.mhlw.go.jp/ shingi/2009/06/dl/s0603-6b.pdf. Accessed 23 Sep 2012.

52. Fowler A, Stodberg T, Eriksson M, Wickstrom R. Childhood encephalitis in Sweden: etiology, clinical presentation and outcome. Eur J Paediatr Neurol. 2008;12:484-90.

53. Hjalmarsson A, Blomqvist $\mathrm{P}$, Brytting $\mathrm{M}$, Linde A, Skoldenberg B. Encephalitis after influenza in Sweden 1987-1998: a rare complication of a common infection. Eur Neurol. 2009;61:289-94.

54. Amin R, Ford-Jones E, Richardson SE, et al. Acute childhood encephalitis and encephalopathy associated with influenza: a prospective 11-year review. Pediatr Infect Dis J. 2008;27:390-5.

55. Ekstrand JJ, Herbener A, Rawlings J, et al. Heightened neurologic complications in children with pandemic H1N1 influenza. Ann Neurol. 2010;68:762-6.

56. Fukumoto Y, Okumura A, Hayakawa F, et al. Serum levels of cytokines and EEG findings in children with influenza associated with mild neurological complications. Brain Dev. 2007;29:425-30.
57. Li WC, Shih SR, Huang YC, et al. Clinical and genetic characterization of severe influenza B-associated diseases during an outbreak in Taiwan. J Clin Virol. 2008;42:45-51.

58. Chung B, Wong V. Relationship between five common viruses and febrile seizure in children. Arch Dis Child. 2007;92:593.

59. Chung BH, Tsang AM, Wong VC. Neurologic complications in children hospitalized with influenza: comparison between USA and Hong Kong. J Pediatr. 2007;151:e17-8.

60. Kwong KL, Lung D, Wong SN, Que TL, Kwong NS. Influenza-related hospitalisations in children. J Paediatr Child Health. 2009;45:660-4.

61. Hon KL, Leung E, Tang J, et al. Premorbid factors and outcome associated with respiratory virus infections in a pediatric intensive care unit. Pediatr Pulmonol. 2008;43:275-80.

62. Louie JK, Gavali S, Acosta M, et al. Children hospitalized with 2009 novel influenza A(H1N1) in California. Arch Pediatr Adolesc Med. 2010;164:1023-31.

63. Parakh A, Kumar A, Kumar V, Dutta AK, Khare S. Pediatric hospitalizations associated with 2009 pandemic influenza A (H1N1): an experience from a tertiary care center in North India. Indian J Pediatr. 2010;77:981-5.

64. Stein M, Tasher D, Glikman D, et al. Hospitalization of children with influenza $\mathrm{A}(\mathrm{H} 1 \mathrm{~N} 1)$ virus in Israel during the 2009 outbreak in Israel: a multicenter survey. Arch Pediatr Adolesc Med. 2010;164:1015-22.

65. Zheng Y, He Y, Deng J, et al. Hospitalized children with 2009 influenza a (H1N1) infection in Shenzhen, China, November-December 2009. Pediatr Pulmonol. 2010; Oct 20. [Epub ahead of print.]

66. Shin SY, Kim JH, Kim HS, et al. Clinical characteristics of Korean pediatric patients critically ill with influenza A (H1N1) virus. Pediatr Pulmonol. 2010;45:1014-20.

67. Zhao C, Gan Y, Sun J. Radiographic study of severe Influenza-A (H1N1) disease in children. Eur J Radiol 2011;79:447-51.

68. Wada T, Morishima T, Okumura A, et al. Differences in clinical manifestations of influenzaassociated encephalopathy by age. Microbiol Immunol. 2009;53:83-8. 
69. Nagao T, Morishima T, Kimura H, et al. Prognostic factors in influenza-associated encephalopathy. Pediatr Infect Dis J. 2008;27:384-9.

70. Wang CC, Chen PY, Wang JD, et al. Clinical and laboratory analysis of influenza B infection in children in Taichung, Taiwan during the 20062007 flu season. Pediatr Neonatol. 2009;50:54-8.

71. Terada K, Kawai Y, Monju A, Wakabayashi T, Ouchi K. Adolescent jump case in Japan associated with influenza but not oseltamivir. Pediatr Infect Dis J. 2008;27:88-9.

72. Takayanagi $\mathrm{M}$, Umehara $\mathrm{N}$, Watanabe $\mathrm{H}$, et al. Acute encephalopathy associated with influenza C virus infection. Pediatr Infect Dis J. 2009;28:554.

73. Okumura A, Abe S, Kidokoro H, Mizuguchi M. Acute necrotizing encephalopathy: a comparison between influenza and non-influenza cases. Microbiol Immunol. 2009;53:277-80.

74. Gika AD, Rich P, Gupta S, Neilson DE, Clarke A. Recurrent acute necrotizing encephalopathy following influenza A in a genetically predisposed family. Dev Med Child Neurol. 2010;52:99-102.

75. Lyon JB, Remigio C, Milligan T, Deline C. Acute necrotizing encephalopathy in a child with H1N1 influenza infection. Pediatr Radiol. 2010;40:200-5.

76. Ng WF, Chiu SC, Lam DS, et al. A 7-year-old boy dying of acute encephalopathy. Brain Pathol. 2010;20:261-4.

77. Troedson C, Gill D, Dale RC. Emergence of acute necrotising encephalopathy in Australia. J Paediatr Child Health. 2008;44:599-601.

78. Neilson DE, Adams MD, Orr CM, et al. Infectiontriggered familial or recurrent cases of acute necrotizing encephalopathy caused by mutations in a component of the nuclear pore, RANBP2. Am J Hum Genet. 2009;84:44-51.

79. Bartynski WS, Upadhyaya AR, Petropoulou KA, Boardman JF. Influenza A encephalopathy, cerebral vasculopathy, and posterior reversible encephalopathy syndrome: combined occurrence in a 3-year-old child. AJNR Am J Neuroradiol. 2010;31:1443-6.

80. Bartynski WS, Upadhyaya AR, Boardman JF. Posterior reversible encephalopathy syndrome and cerebral vasculopathy associated with influenza A infection: report of a case and review of the literature. J Comput Assist Tomogr. 2009;33:917-22.
81. Henry J, Smeyne RJ, Jang H, Miller B, Okun MS. Parkinsonism and neurological manifestations of influenza throughout the 20th and 21st centuries. Parkinsonism Relat Disord. 2010;16:566-71.

82. Toovey S, Jick SS, Meier CR. Parkinson's disease or Parkinson symptoms following seasonal influenza. Influenza Other Respi Viruses. 2011;5:328-33.

83. Hoffmann G, Funk C, Fowler $S$, et al. Nonclinical pharmacokinetics of oseltamivir and oseltamivir carboxylate in the central nervous system. Antimicrob Agents Chemother. 2009;53:4753-61.

84. Hatori A, Arai T, Yanamoto $\mathrm{K}$, et al. Biodistribution and metabolism of the antiinfluenza drug [11C]oseltamivir and its active metabolite [11C]Ro 64-0802 in mice. Nucl Med Biol. 2009;36:47-55.

85. Jhee SS, Yen M, Ereshefsky L, et al. Low penetration of oseltamivir and its carboxylate into cerebrospinal fluid in healthy Japanese and Caucasian volunteers. Antimicrob Agents Chemother. 2008;52:3687-93.

86. Ose $\mathrm{A}$, Ito $\mathrm{M}$, Kusuhara $\mathrm{H}$, et al. Limited brain distribution of [3R,4R,5S]-4-acetamido-5-amino3-(1-ethylpropoxy)-1-cyclohexene-1-carboxylate phosphate (Ro 64-0802), a pharmacologically active form of oseltamivir, by active efflux across the blood-brain barrier mediated by organic anion transporter 3 (Oat3/Slc22a8) and multidrug resistance-associated protein 4 (Mrp4/ Abcc4). Drug Metab Dispos. 2009;37:315-21.

87. Uchida Y, Ohtsuki S, Katsukura Y, et al. Quantitative targeted absolute proteomics of human blood-brain barrier transporters and receptors. J Neurochem. 2011;117:333-45.

88. Oshima S, Nemoto E, Kuramochi M, Saitoh Y, Kobayashi D. Penetration of oseltamivir and its active metabolite into the brain after lipopolysaccharide-induced inflammation in mice. J Pharm Pharmacol. 2009;61:1397-400.

89. Lindemann L, Jacobsen H, Schuhbauer D, et al. In vitro pharmacological selectivity profile of oseltamivir prodrug (Tamiflu ${ }^{\circledR}$ ) and active metabolite. Eur J Pharmacol. 2010;628:6-10.

90. Izumi Y, Tokuda K, O’Dell KA, Zorumski CF, Narahashi T. Neuroexcitatory actions of Tamiflu and its carboxylate metabolite. Neurosci Lett. 2007;426:54-8. 
91. Knoflach F, Trube G, Buisson B, Steidl-Armougom E, Prinssen P, Lindemann L. Evaluation of pharmacological activities of oseltamivir on paired-pulse inhibition in the CA1 region of adult rat hippocampus. p. 822.18. Available at: http:// www.sfn.org/skins/main/pdf/abstracts/am2009/ poster_presentations/PosterPresentation_WedPM. pdf. Accessed Sep 102012.

92. Usami A, Sasaki T, Satoh N, et al. Oseltamivir enhances hippocampal network synchronization. J Pharmacol Sci. 2008;106:659-62.

93. Satoh K, Nonaka R, Ogata A, Nakae D, Uehara S. Effects of oseltamivir phosphate (Tamiflu) and its metabolite (GS4071) on monoamine neurotransmission in the rat brain. Biol Pharm Bull. 2007;30:1816-8.

94. Data on file. Basel: Roche; 1998.

95. Izumi Y, Tokuda K, O’Dell K, Zorumski C, Narahashi T. Synaptic and behavioral interactions of oseltamivir (Tamiflu) with neurostimulants. Hum Exp Toxicol. 2008;27:911-7.

96. Uchimura N, Kuwahara H, Kumagai $\mathrm{Y}$, et al. Absence of adverse effects of oseltamivir on sleep: a double-blind, randomized study in healthy volunteers in Japan. Basic Clin Pharmacol Toxicol. 2011;109:309-14.

97. Doucette KE, Aoki FY. Oseltamivir: a clinical and pharmacological perspective. Expert Opin Pharmacother. 2001;2:1671-83.

98. He G, Massarella J, Ward P. Clinical pharmacokinetics of the prodrug oseltamivir and its active metabolite Ro 64-0802. Clin Pharmacokinet. 1999;37:471-84.

99. Shi D, Yang J, Yang D, et al. Anti-influenza prodrug oseltamivir is activated by carboxylesterase human carboxylesterase 1 , and the activation is inhibited by antiplatelet agent clopidogrel. J Pharmacol Exp Ther. 2006;319:1477-84.

100. Zhu HJ, Markowitz JS. Activation of the antiviral prodrug oseltamivir is impaired by two newly identified carboxylesterase 1 variants. Drug Metab Dispos. 2009;37:264-7.

101. Yang D, Pearce RE, Wang X, et al. Human carboxylesterases HCE1 and HCE2: ontogenic expression, inter-individual variability and differential hydrolysis of oseltamivir, aspirin, deltamethrin and permethrin. Biochem Pharmacol. 2009;77:238-47.

102. Zhu HJ, Appel DI, Jiang Y, Markowitz JS. Ageand sex-related expression and activity of carboxylesterase 1 and 2 in mouse and human liver. Drug Metab Dispos. 2009;37:1819-25.
103. Oo C, Barrett J, Hill G, et al. Pharmacokinetics and dosage recommendations for an oseltamivir oral suspension for the treatment of influenza in children. Paediatr Drugs. 2001;3:229-36.

104. Acosta EP, Jester P, Gal P, et al. Oseltamivir dosing for influenza infection in premature neonates. J Infect Dis. 2010;202:563-6.

105. Aiba H, Mochizuki M, Kimura M, Hojo $H$. Predictive value of serum interleukin-6 level in influenza virus-associated encephalopathy. Neurology. 2001;57:295-9.

106. Ichiyama $\mathrm{T}$, Isumi $\mathrm{H}$, Ozawa $\mathrm{H}$, et al. Cerebrospinal fluid and serum levels of cytokines and soluble tumor necrosis factor receptor in influenza virusassociated encephalopathy. Scand J Infect Dis. 2003;35:59-61.

107. Cisse Y, Wang S, Inoue I, Kido H. Rat model of influenza-associated encephalopathy (IAE): studies of electroencephalogram (EEG) in vivo. Neuroscience. 2010;165:1127-37.

108. Tanaka T, Sunden Y, Sakoda Y, et al. Lipopolysaccharide treatment and inoculation of influenza A virus results in influenza virusassociated encephalopathy-like changes in neonatal mice. J Neurovirol. 2010;16:125-32.

109. Riazi K, Galic MA, Kuzmiski JB, et al. Microglial activation and TNFalpha production mediate altered CNS excitability following peripheral inflammation. Proc Natl Acad Sci USA. 2008;105:17151-6.

110. Tsuge $\mathrm{M}$, Yasui $\mathrm{K}$, Ichiyawa $\mathrm{T}$, et al. Increase of tumor necrosis factor-alpha in the blood induces early activation of matrix metalloproteinase- 9 in the brain. Microbiol Immunol. 2010;54:417-24.

111. Serrats J, Schiltz JC, Garcia-Bueno B, et al. Dual roles for perivascular macrophages in immune-tobrain signaling. Neuron. 2010;65:94-106.

112. Holmes C, Cunningham C, Zotova E, et al. Systemic inflammation and disease progression in Alzheimer disease. Neurology. 2009;73:768-74.

113. Steinman L. Nuanced roles of cytokines in three major human brain disorders. J Clin Invest. 2008;118:3557-63.

114. Raison CL, Capuron L, Miller AH. Cytokines sing the blues: inflammation and the pathogenesis of depression. Trends Immunol. 2006;27:24-31.

115. Bentivoglio M, Kristensson K. Neural-immune interactions in disorders of sleep-wakefulness organization. Trends Neurosci. 2007;30:645-52. 
116. Matsubara K, Kodera M, Nigami H, Yura K, Fukaya T. Reversible splenial lesion in influenza virus encephalopathy. Pediatr Neurol. 2007;37:431-4.

117. Fluss J, Ferey S, Menache-Starobinski C, et al. Mild influenza-associated encephalopathy/encephalitis with a reversible splenial lesion in a Caucasian child with additional cerebellar features. Eur J Paediatr Neurol. 2010;14:97-100.

118. Ganapathy S, Ey EH, Wolfson BJ, Khan N. Transient isolated lesion of the splenium associated with clinically mild influenza encephalitis. Pediatr Radiol. 2008;38:1243-5.

119. Iwata A, Matsubara K, Nigami H, Kamimura K, Fukaya T. Reversible splenial lesion associated with novel influenza A (H1N1) viral infection. Pediatr Neurol. 2010;42:447-50.

120. Tsuji M, Yoshida T, Miyakoshi C, Haruta T. Is a reversible splenial lesion a sign of encephalopathy? Pediatr Neurol. 2009;41:143-5.

121. Kotan D. Widespread subarachnoidal pneumocephalus development as a complication of influenza: a case report. Arch Med Sci. 2008;4:471-3.

122. Sumikoshi M, Hashimoto K, Kawasaki Y, et al. Human influenza virus infection and apoptosis induction in human vascular endothelial cells. J Med Virol. 2008;80:1072-8.

123. Wang G, Zhang J, Li W, et al. Apoptosis and proinflammatory cytokine responses of primary mouse microglia and astrocytes induced by human H1N1 and avian H5N1 influenza viruses. Cell Mol Immunol. 2008;5:113-20.

124. Kawada J, Kimura H, Ito Y, et al. Systemic cytokine responses in patients with influenza-associated encephalopathy. J Infect Dis. 2003;188:690-8.
125. van Zeijl JH, Bakkers J, Wilbrink B, et al. Influenzaassociated encephalopathy: no evidence for neuroinvasion by influenza virus nor for reactivation of human herpesvirus 6 or 7 . Clin Infect Dis. 2005;40:483-5.

126. Morishima T. Influenza-associated encephalopathy. No To Shinkei. 2006;58:561-9. In Japanese.

127. Shinohara M, Saitoh M, Takanashi JI, et al. Carnitine palmitoyl transferase II polymorphism is associated with multiple syndromes of acute encephalopathy with various infectious diseases. Brain Dev. 2011;33:512-7.

128. Yao D, Mizuguchi H, Yamaguchi M, et al. Thermal instability of compound variants of carnitine palmitoyltransferase II and impaired mitochondrial fuel utilization in influenza-associated encephalopathy. Hum Mutat. 2008;29:718-27.

129. Chen Y, Mizuguchi H, Yao D, et al. Thermolabile phenotype of carnitine palmitoyltransferase II variations as a predisposing factor for influenza-associated encephalopathy. FEBS Lett. 2005;579:2040-4.

130. Hosoya M, Kawasaki Y, Katayose M, et al. Prognostic predictive values of serum cytochrome c, cytokines, and other laboratory measurements in acute encephalopathy with multiple organ failure. Arch Dis Child. 2006;91:469-72.

131. Purevsuren J, Hasegawa Y, Kobayashi H, Endo M, Yamaguchi S. Urinary organic metabolite screening of children with influenza-associated encephalopathy for inborn errors of metabolism using GC/MS. Brain Dev. 2008;30:520-6.

132. Okamoto E. Incidence of injuries after influenza using health insurance claims. Jpn J Pharmacoepidemiol. 2009;21:50-1.

133. Berger JR, Houff SA. Neurological infections: the year of PML and influenza. Lancet Neurol. 2010;9:14-7. 